\title{
Плотность и вязкость растворов хладагент R600a / минеральное масло / фуллерены $\mathrm{C}_{60}$
}

\author{
С. А. Мороз, Н. Н. Лукьянов, В. П. Железный
}

Одесская национальная академия пищевых технологий, ул. Канатная 112, г. Одесса, 65039, Украина

В статье представлены результаты экспериментального исследования температурной и концентрационной зависимостей плотности и вязкости, растворов хладагент R600a/минеральное масло ХФ16-12/ фуллерены C 60 . Измерения плотности выполнены пикнометрическим методом в диапазоне температур 258-353 К. Вязкость измерена методом катящегося шарика в интервале температур 253-283 К. На основании полученных экспериментальных данных выполнен анализ влияния примесей компрессорного масла и фуллеренов $\mathrm{C}_{60}$ на плотность и вязкость хладагента R600a. Показано, что примеси фуллеренов способствуют понижению вязкости рабочего тела (хладагент R600a/минеральное масло ХФ16-12) во всем интервале параметров исследования. Рабочее тело R600a/минеральное масло ХФ16-12/ фуллерены $\mathrm{C}_{60}$ рекомендуется для использования в бытовой холодильной технике.

Ключевые слова: Растворы; Хладагент; Фуллерены; Эксперимент; Плотность; Вязкость; Концентрация

\section{Густина і в'язкість розчинів холодоагент R600a / мінеральне масло / фулерени $\mathrm{C}_{60}$}

\section{С. О. Мороз, М. М. Лук'янов, В. П. Желєзний}

Одеська національна академія харчових технологій, вул. Канатна 112, м. Одеса, 65039, Україна

У статті представлені результати експериментального дослідження температурної і концентраційної залежності густини і в'язкості, розчинів холодоагент R600a / мінеральне масло ХФ16-12 / фулерени С 60 . Вимірювання густини виконані пікнометричним методом в діапазоні температур 258-353 К. В'язкість виміряна методом кульки, що котиться в інтервалі температур 253-283 К. Робоче тіло - R600a / мінеральне масло ХФ16-12 / фулерени C 60 рекомендується для використання в побутовій холодильній техніці. На підставі отриманих експериментальних даних виконано аналіз впливу домішок компресорного масла і фулеренів $\mathrm{C}_{60}$ на густину і в'язкість холодоагенту R600a. Показано, що домішки фулеренів сприяють зниженню в'язкості робочого тіла холодоагент R600a / мінеральне масло ХФ16-12 у всьому інтервалі параметрів дослідження.

Ключові слова: Розчини; Холодоагент; Фулерени; Експеримент; Густина; В'язкість; Концентрація

DOI: http://dx.doi.org/10.15673/ret.v53i1.544

This is an open access article under the Creative Commons Attribution 4.0 International License (CC BY) http://creativecommons.org/licenses/by/4.0/

C The Author(s) 2017.

\section{1 Введение}

В настоящее время включение наночастиц в состав рабочего тела компрессионной холодильной системы рассматривается как один из перспективных методов повышения показателей эффективности холодильной техники [1]. Наиболее часто наночастицы добавляют в компрессорные масла, которые в свою очередь являются необходимым компонентом реального рабочего тела (РРТ) в парокомпрессионном холодильном оборудовании [1-3]. Ряд авторов [1-4] отмечают, что повышение показателей энергетической эффективности холодильного оборудования достигается как за счет изменения теплофизиче- ских свойств реального рабочего тела (раствора хладагент/масло (РХМ), так и уменьшения трения в сопрягаемых деталях компрессорной системы и интенсификации процессов теплообмена в аппаратах холодильного оборудования.

Проблема учета влияния наночастиц на вязкость технических жидкостей рассматривалась во многих работах. Так авторы статьи [5] привели результаты исследования вязкости $\mathrm{TiO}_{2} /$ этиленгликоль, при концентрациях 0,5-8,0 масс. \% и температуре $20-60^{\circ} \mathrm{C}$. Приведенные данные показывают, что вязкость раствора увеличилась с увеличением концентрации наночастиц. Вязкость изученных растворов сильно зависела от температуры и концентрации наночастиц. 
В работе [6] авторы представили результаты исследования влияния сурфактантов SDBS на вязкость растворов $\mathrm{Al}_{2} \mathrm{O}_{3}$ /вода при концентрациях $\mathrm{Al}_{2} \mathrm{O}_{3}$ 0,04-0,16 масс. \% и температурах $30-70^{\circ} \mathrm{C}$. Результаты этих исследований показывают, что основной эффект на вязкость оказывают сурфактанты и температура. Напротив, влияние наночастиц на вязкость изученных растворов было незначительным.

Авторы работы [7] представили данные о вязкости нанофлюидов $\mathrm{SiO}_{2} /$ вода и $\mathrm{Al}_{2} \mathrm{O}_{3} /$ вода. Результаты этого исследования показывают, что вязкость изученных растворов увеличилась с возрастанием концентрации наночастиц.

Рудяк В.Я. в работе [8] приводит результаты исследования вязкости на основе дистиллированной воды с частицами оксида меди. Приведенные данные показывают, с уменьшением размера наночастиц вязкость нанофлюидов увеличивается. Причем, при объемной концентрации наночастиц оксида меди 0,25\%, жидкость становиться неньютоновской.

Ряд исследователей отмечает, что выбор базовой жидкости не имеет существенного влияния на относительное изменение вязкости нанофлюидов. Например, нанофлюиды $\mathrm{Al}_{2} \mathrm{O}_{3}$ /этиленгликоль и $\mathrm{Al}_{2} \mathrm{O}_{3}$ /вода имеют аналогичный прирост вязкости базовых жидкостей [9].

Этот вывод не является бесспорным. Например, авторы работы [10] обнаружили различный прирост вязкости для двух вышеуказанных наножидкостей.

Данные о влиянии наночастиц на вязкость базовых жидкостей носят очень противоречивый характер. Так, например, в статьях [9] и [11] авторы обнаружили близкие значения увеличения вязкости для водных растворов, содержащих 5 об. \% $\mathrm{Al}_{2} \mathrm{O}_{3}$. Напротив, в работе [12, 13] авторы обнаружили различный прирост вязкости для одного и того же типа наножидкостей. В работе [14] авторы отмечают, что вязкость наножидкостей сильно зависит от свойств базовой жидкости, но слабо зависит от материала наночастиц.

Некоторые авторы в своих работах [15-17] показывают, что относительная вязкость не зависит от температуры. Ряд авторов, например, [18-20] отмечают, что вязкость наножидкостей уменьшается нелинейно или экспоненциально с увеличением температуры.

С последним выводом не согласны некоторые авторы. Например, ряд исследователей $[15,21]$ указывают на линейное увеличение вязкости с увеличением объемных концентраций наночастиц. Напротив, другие авторы [16, 22, 23] отмечают нелинейный характер изменения вязкости от концентрации наночастиц. Причем, для одних тех же наножидкостей с одинаковой концентрацией наночастиц иногда проявляется разное увеличение вязкости.

Среди авторов, которые изучают вязкость нанофлюидов, отсутствует общее мнение о влиянии размера частиц на вязкость наножидкостей. Некоторые исследователи сообщают, что размер частиц не оказывает существенного влияния на вязкость [15]. Однако большая часть исследователей отмечают, что размер наночастиц и их форма оказывают заметное влияние на вязкость базовых жидкостей. Например, авторы работ
[19, 24-26] утверждают, что вязкость нанофлюидов увеличивается с уменьшением размера наночастиц. Напротив, другие авторы [22, 23, 27] считают, что имеется обратная тенденция изменения вязкости за счет увеличения размера частиц.

Следует заметить, что в литературе практически отсутствует информация о влиянии наночастиц на плотность и вязкость реальных рабочих тел для компрессионных холодильных машин [28]. Вместе с тем теплофизические свойства нанофлюидов, которые получены на основе растворов хладагент/масло, необходимы для изучения процессов теплообмена при конденсации и кипении рабочего тела в испарителе холодильной машины.

В ряде публикаций, например [29], авторы отмечают, что процессы теплообмена зависят от присутствия наночастиц в рабочем теле, которые влияют на теплофизические свойства базовых жидкостей, в том числе и на вязкость $[29,30]$. Причем увеличение коэффициента теплоотдачи в испарителе обусловлено не только изменениями теплофизических свойств кипящего нанохладагента, но и за счет образования дополнительных центров парообразования [31].

В литературе уже представлено несколько обзоров $[30,32,33]$, в которых изучается влияние наночастиц на вязкость базовых жидкостей. Большинство авторов отмечают, что примеси наночастиц способствуют повышению вязкости базовых жидкостей. Причем вязкость нанофлюидов зависит как от температуры и объемной концентрации, так и размеров и формы наночастиц

Однако в литературе до сих пор отсутствуют публикации, посвященные изучению влияния фуллеренов на вязкость рабочих тел. Очевидно, что без достоверных данных по вязкости рабочих тел с примесями наночастиц достаточно сложно интерпретировать экспериментальные данные как по теплообмену в испарителе, так и по затратам энергии на работу компрессора холодильной машины [1].

Кроме того, исследования вязкости растворов хладагент/масло/наночастицы должны способствовать формированию базы справочных данных о теплофизических свойствах технически важных нанофлюидов. В настоящее время в литературе отсутствуют надежные модели для прогнозирования вязкости нанофлюидов [32]. Поэтому формирование базы справочных данных о теплофизических свойствах нанофлюидов будет способствовать разработке научно обоснованных представлений о взаимодействии наночастиц с молекулами базовой жидкости, которые лягут в основу новых моделей прогнозирования свойств нанофлюидов.

Изучение теплофизических свойств нанофлюидов на основе рабочих тел парокомпрессионных холодильных машин является актуальной проблемой, которая требует своего решения. Именно термодинамические и транспортные свойства рабочих тел определяют, как величину показателей эффективности холодильной компрессорной системы, так и интенсивность процессов теплообмена в аппаратах холодильных установок. 


\section{2 Подготовка образцов}

Анализ опубликованных работ, посвященных изучению вязкости нанофлюидов [32, 33], показывает, что подготовка образцов является, ключевым фактором для получения стабильных образцов для проведения исследований.

В настоящей работе компонентами объектов исследования являлись: хладагент R600a (изобутан), компрессорное минеральное масло ХФ16-12 (вязкость при $40^{\circ} \mathrm{C} 16 \mathrm{~mm}^{2} / \mathrm{c}$ ) и фуллерены $\mathrm{C}_{60}$ (Sigma Aldrihc) с размером не более 0,7 нм.

Исследование вязкости раствора изобутана R600a, минерального масла ХФ 16-12 с примесями фуллеренов $C_{60}\left(w_{\text {nапо }}=0,2\right.$ масс. \% в масле) проводились при трех концентрациях масла в ROS: 0,25, 0,5 и 0,75 масс.\%. Технология приготовления наномасла заключалась в диспергировании наночастиц $\mathrm{C}_{60}$ в компрессорном масле в ультразвуковой ванне Codison CD 4800 с частотой 42 кГц в течение 30 минут. Длительное (в течении 1 месяца) наблюдение за компрессорным маслом с добавками наночастиц (наномаслом) показало хорошую агрегативную устойчивость фуллеренов (без выпадения осадка и помутнения). Для приготовления рабочего тела R600a/XФ16-12/ 6 бо определенное количество наномасла смешивалось с необходимым количеством хладагента без дополнительного ультразвукового диспергирования.

\section{3 Определение плотности растворов хладагент R600a / минеральное компрессорное масло / фуллерены $\mathrm{C}_{60}$}

Исследование плотности РХМ производилось пикнометрическим методом. Схема экспериментальной установки по исследованию плотности объектов исследования приведена на рисунке 1. Стеклянная ампула с исследуемым веществом 3 (пикнометр) помещена в термостат 4, оснащенный теплообменником 2, по которому прокачивается термостатирующая жидкость из термостата 8. При измерении плотности при температурах ниже окружающей среды термостат 8 подключался к холодильной машине (на рисунке 1 не показано). Проведенные исследования показывают, что колебания температуры в термостате 4 не превышали 0,02 К. Измерение сопротивления термометра осуществлялось по компенсационной схеме с использованием мультиметра 7. Температура в проведенных экспериментах измерялась платиновым термометром сопротивления 6 (Wika TR10A) с расширенной неопределенностью не более $\pm 0,05$ K). Расширенная неопределённость измерения концентрации примесей масла в РХМ не превышала 0,04\% и концентрации фуллеренов в масле не более $1,47 \%$.

Для измерения уровня жидкой фазы образца РХМ в измерительной ячейке (пикнометре) использовался катетометр 5.

Эксперимент проводился в интервале температур от 258 до $353 \mathrm{~K}$ и массовых долях хладагента в масле: 0,25; 0,5 и 0,75 кг/кг. Расширенная неопределенность измерения плотности РХМ не превышала 6 кг/м³. Влияние наночастиц на плотность РХМ рассчитывалось по методике, приведенной в работе [33].

$$
\rho_{\text {nanoROS }}=\varphi_{\text {nano }} \cdot \rho_{\text {nano }}+\left(1-\varphi_{\text {nano }}\right) \cdot \rho_{\text {ROS }},
$$

где $\varphi_{\text {nano }}$ - объемная доля фуллеренов в растворе, $\mathrm{M}^{3} / \mathrm{M}^{3} ; \rho_{\text {nano }}-$ плотность фуллеренов, кг $/ \mathrm{M}^{3} ; \rho_{\text {ROS }}-$ плотHOCTь ROS, $\mathrm{Kr} / \mathrm{M}^{3}$.

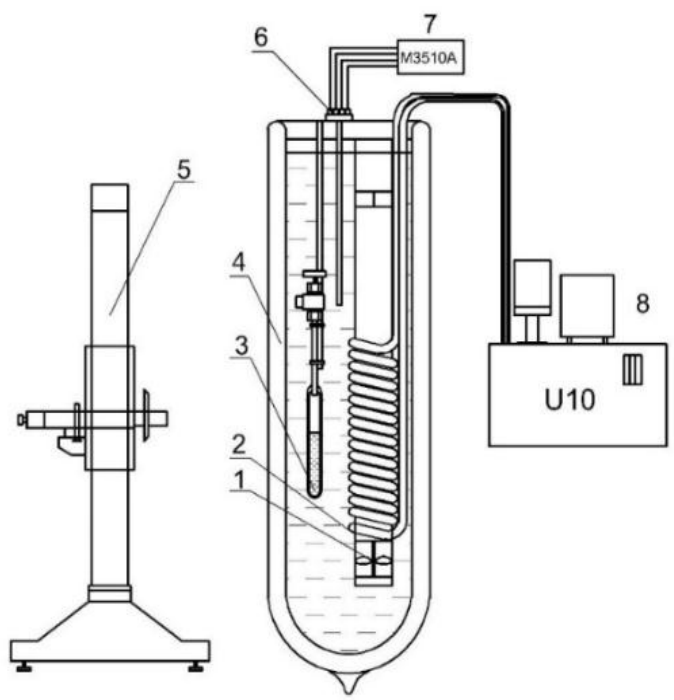

Рисунок 1 - Экспериментальная установка по исследованию плотности РХМ: 1 - мешалка; 2 - теплообменник; 3 - ячейка с исследуемым веществом; 4 термостат; 5 - катетометр; 6 - платиновый термометр сопротивления TR10A; 7 - мультиметр M3510A; 8- термостат U10

Результаты проведенного исследования приведены в таблице 1. Концентрационную зависимость плотности растворов R600a/минеральное масло при различных температурах демонстрирует рисунок 2.

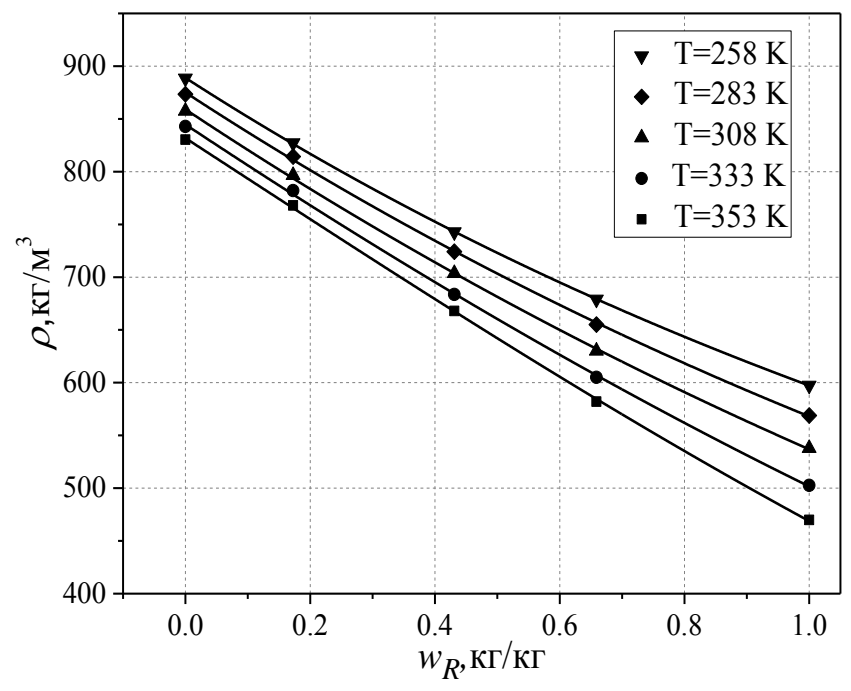

Рисунок 2 - Концентрационная зависимость плотности растворов R600а/минеральное масло при различных температурах 
Таблица 1 - Экспериментальные данные плотности и вязкости растворов R600a/XФ16-12 и R600a/XФ16-12/C60

\begin{tabular}{|c|c|c|c|c|c|c|c|c|c|}
\hline \multicolumn{3}{|c|}{$\begin{array}{c}\text { Плотность раствора } \\
\text { R600a/масло } \\
\end{array}$} & \multicolumn{3}{|c|}{$\begin{array}{c}\text { Динамическая вязкость раствора } \\
\text { R600a/масло }\end{array}$} & \multicolumn{4}{|c|}{$\begin{array}{c}\text { Динамическая вязкость раствора } \\
\text { PXM/C } 6 \text { ( }\end{array}$} \\
\hline$T, \mathrm{~K}$ & $\rho, \kappa г / \mathrm{M}^{3}$ & $\begin{array}{c}w_{R}, \\
\text { мacc. } \%\end{array}$ & $T, \mathrm{~K}$ & $\eta, \mathrm{м} \Pi а \cdot \mathrm{c}$ & $\begin{array}{c}w_{R}, \\
\text { мacc. } \%\end{array}$ & $T, \mathrm{~K}$ & $\begin{array}{c}\eta, \\
\mathrm{м} \Pi \mathrm{a} \cdot \mathrm{c}\end{array}$ & $\begin{array}{c}w_{R}, \\
\text { мacc.\% }\end{array}$ & $\begin{array}{c}w_{C 60}, \\
\text { масс. } \%\end{array}$ \\
\hline 258,32 & 679,4 & \multirow{5}{*}{65,8} & 255,18 & 1,99 & \multirow{4}{*}{74,9} & 255,18 & 1,95 & \multirow{4}{*}{74,3} & \multirow{4}{*}{0,05} \\
\hline 283,21 & 655,3 & & 265,15 & 1,82 & & 265,15 & 1,79 & & \\
\hline 308,12 & 630,5 & & 275,10 & 1,69 & & 275,11 & 1,66 & & \\
\hline 333,24 & 605,1 & & 285,20 & 1,55 & & 285,28 & 1,53 & & \\
\hline 353,22 & 582,2 & & & & & & & & \\
\hline 258,15 & 742,6 & \multirow{5}{*}{43,1} & 255,18 & 3,01 & \multirow{4}{*}{47,9} & 255,18 & 2,93 & \multirow{4}{*}{47,9} & \multirow{4}{*}{0,1} \\
\hline 283,12 & 724,2 & & 265,15 & 2,76 & & 265,15 & 2,70 & & \\
\hline 308,13 & 703,4 & & 275,10 & 2,54 & & 275,12 & 2,49 & & \\
\hline 333,32 & 683,3 & & 285,20 & 2,37 & & 285,23 & 2,34 & & \\
\hline 353,23 & 668,2 & & & & & & & & \\
\hline 258,22 & 827,5 & \multirow{5}{*}{17,2} & 255,18 & 5,03 & \multirow{4}{*}{24,9} & 255,18 & 4,85 & \multirow{4}{*}{24,9} & \multirow{4}{*}{0,15} \\
\hline 283,11 & 814,3 & & 265,15 & 4,49 & & 265,15 & 4,36 & & \\
\hline 308,33 & 796,1 & & 275,10 & 4,00 & & 275,15 & 3,89 & & \\
\hline 333,14 & 782,2 & & 282,50 & 3,75 & & 285,24 & 3,63 & & \\
\hline 353,21 & 768,2 & & & & & & & & \\
\hline
\end{tabular}

4 Измерение вязкости растворов хладагент R600a / минеральное компрессорное масло / фуллерены $\mathrm{C}_{60}$

Экспериментальное исследование динамической вязкости - $\eta$ растворов хладагент/масло и хладагент/масло/фуллерены C $_{60}$ выполнено методом катящегося шарика на вискозиметре Гепплера. Схема экспериментальной установки представлена на рисунке 3.

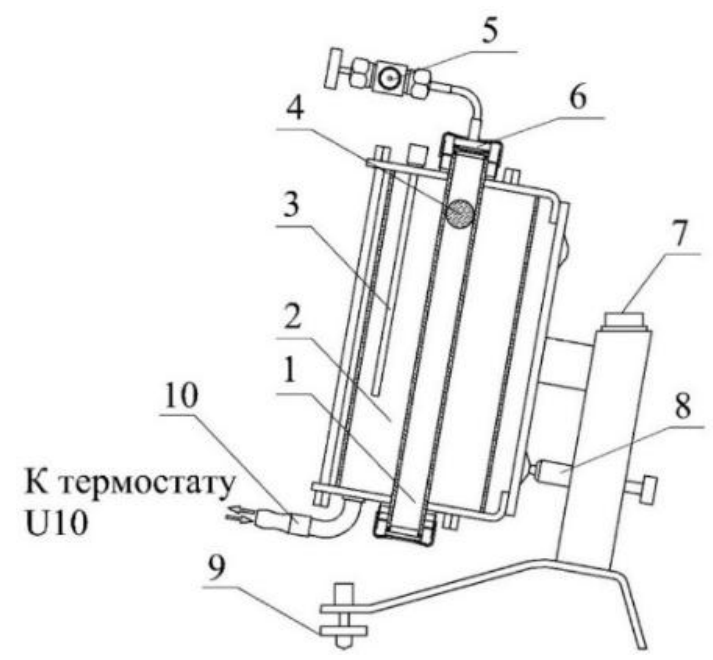

Рисунок 3 - Экспериментальная установка для исследования динамической вязкости РХМ и наноРХМ методом катящегося шарика:

1 - трубка с исследуемым веществом; 2 - термостат; 3 - платиновый термометр сопротивления TEPA 500 Pt; 4 - шарик; 5 - заправочный вентиль; 6 крышка; 7 - уровень; 8 - фиксирующий шток; 9 винт для установки уровня; 10 - патрубок для соединения с термостатом.
Измерения вязкости выполнены в диапазоне температур от 253 до 293 K и массовых долях хладагента в масле: для ROS 0,25, 0,5 и 0,75 кг/кг, для $\mathrm{ROS} / \mathrm{C}_{60}$ 0,25, 0,5 и 0,75. Концентрация фуллеренов $\mathrm{C}_{60}$ в масле составляла 0,2 масс.\%. Расширенная неопределенность измерения температуры в проведённых исследованиях не превышала 0,2К, концентрации примесей масла в ROS 0,04 \% и примесей фуллеренов в масле \pm 1.47 \%. Результаты экспериментальных данных приведены в таблице 1. Зависимость динамической вязкости растворов R600a/XФ16-12 и R600a/ХФ16$12 / C_{60}$ ( $w_{\text {nano }}=0,2$ масс. \% в масле) от концентрации хладагента в жидкой фазе $-w_{R}$. представлена рисунке 4.

Расчет динамической вязкости РХМ и наноРХМ производился по следующей формуле

$$
\eta=K \cdot\left(\rho_{b}-\rho^{\prime}\right) \cdot \tau,
$$

где $K$ - постоянная прибора, $\mathrm{m}^{2} / \mathrm{c}^{2} ; \rho_{b}$ - плотность ша-

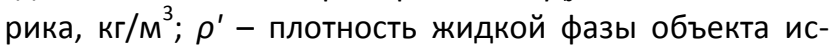
следования, кг $/ \mathrm{m}^{3} ; \tau$ - время перемещения шарика на заданном участке, с.

Выполненный анализ показывает, что расширенная неопределенность измерения вязкости PXM и наноРХМ в проведенных исследованиях в зависимости от диапазона находится при температуре $253 \mathrm{~K}$ равна 0.02 мПа·с и при температуре 283K-0.88 мПа·с.

\section{5 Заключение}

Проведенное исследования плотности и вязкости растворов R600a ХФ16-12 и R600a/ХФ16-12/C 60 показывает, что примеси компрессорного масла в хладагенте R600a приводят к значительному увеличению плотности и вязкости. Поскольку концентрация фуллеренов очень незначительна (см. таблицу) их влияние на плотность практически отсутствует. Напротив, влияние фуллеренов на вязкость РХМ представляет значительный интерес. 


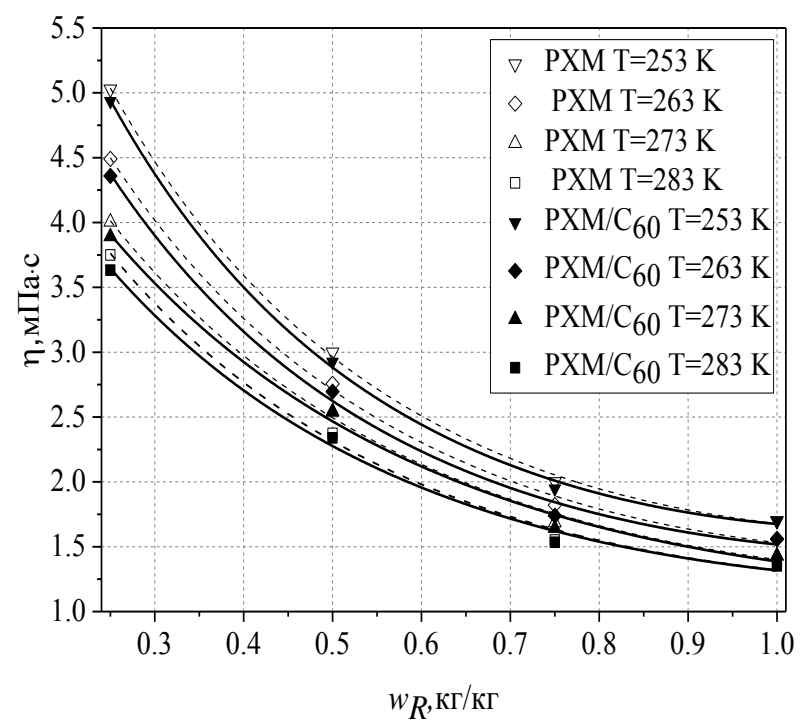

Рисунок 4 - Зависимость динамической вязкости pacmвopa R600a XФ16-12 u R600a/ХФ16-12/C 60 ( $w_{\text {nапо }}=0,2$ масс. \% в масле) от концентрации хладагента в жидкой фразе $-w_{R}$

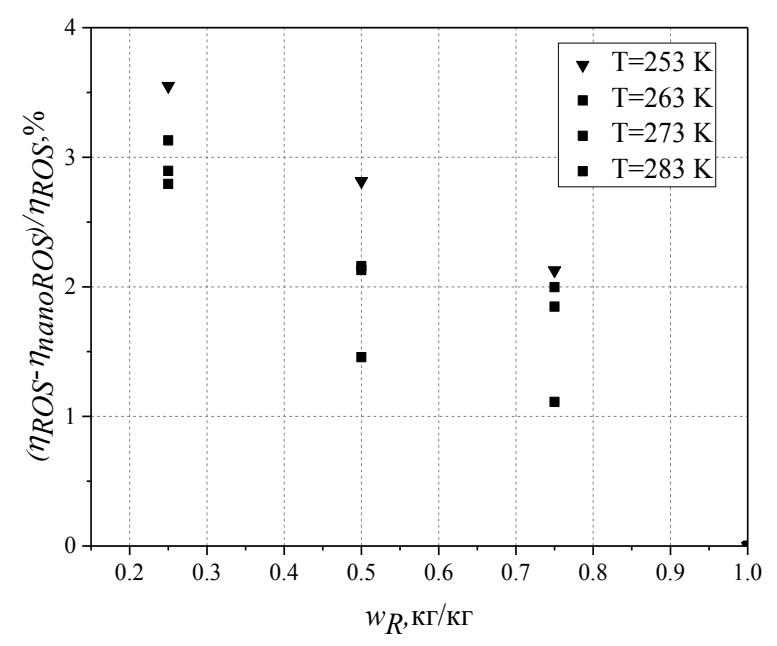

Рисунок 5 - Зависимость относительных отклонений $\left(\eta_{\text {ROS }}-\eta_{\text {nanoROS }}\right) / \eta_{\text {PXM }}$, для растворов R600a/минеральное масло и R600a/ минеральное масло / $\mathrm{C}_{60} \quad\left(\mathrm{w}_{\text {nano }}=0,2\right.$ масс \% в масле) от концентрации хладагента в жидкой фазе $-\mathrm{w}_{\mathrm{R}}$

Проведенные исследования показывают, что даже небольшие примеси наночастиц в рабочем теле парокомпрессионной холодильной машины приводят к значительному уменьшению вязкости РХМ - от 1\% до $3,5 \%$. Этот эффект влияния фуллеренов несомненно имеет позитивное значение. Из полученных экспериментальных данных следует, что уменьшение затрат электроэнергии потребляемой компрессорной системой $[1,34]$ определяются как уменьшением трения в сопрягаемых деталях компрессора, так и уменьшением вязкости РХМ. Уменьшение вязкости растворов хладагент/масло будет также способствовать лучшему уносу примесей масла их испарителя холодильной машины. С учетом высокой стабильности нанофлюидов, можно констатировать, что применение примесей фуллеренов в рабочем теле имеет высокую перспективу внедрения в парокомпрессионной бытовой холодильной технике.

\section{Литература}

1. Мороз, С.А. Экспериментальное исследование влияния примесей фуллеренов $\mathrm{C}_{60}$ В компрессорном масле и величины вязкости масла на показатели эффективности холодильной компрессорной системы [Текст] / С.А.Мороз, О.Я. Хлиева, Н.Н. Лукьянов, В.П. Железный // Вестник Международной академии холода. 2016. - №41. - C. 41-46.

2. Kedzierski, M.A. Effect of CuO Nanoparticle Concentration on R134a-Lubricant Pool Boiling Heat Transfer [Text] / M.A. Kedzierski // Micro-Nanoscale Heat Transfer International Conference, 2008: Proceedings Taiwan - N. Y. P. 1-8.

3. Azmi, M. Z. Potential of nanorefrigerant and nanolubricant on energy saving in refrigeration system [Text] / W. H. Azmi, M. Z. Sharif, T. M. Yusof // Renew. Sust. Energ. Rev. - 2017. -Vol. Issue 69. - P. 415-428.

4. Wang, R. Use of nanoparticles to make mineral oil lubricants feasible for use in a residential air conditioner employing hydro-fluorocarbons refrigerants [Text] / R. Wang, Q. Wu, Y. Wu // Energy and Buildings. - 2010. Vol. 42, Issue 11. - P. 2111-2117.

5. Chen, $\mathbf{H}$. Rheological behavior of nanofluids [Text] / $\mathrm{H}$. Chen, Y. Ding, C. Tan. // New Journal of Physics. - 2007. Vol. 9, Issue 367.P. -24 .

6. Zhu, D.S. Influence of SDBS on viscosity for aluminium nano-suspensions [Text] / D.S. Zhu, X.F. Li, J.W. Gao, X.J. Wang, H. Li, Z.J. Wu // International Congress of Refrigeration. - 2007. -P.1- 7.

7. Tavman, I. Experimental investigation of viscosity and thermal conductivity of suspensions containing nanosized ceramic particles [Text] / I. Tavman, A. Turgut, M. Chirtoc, H.P. Schuchmann, S. Tavman // International Scientific Journal. - 2008. - Vol.34, Issue 2. - P. 99-104.

8. Гузей, Д. В. Измерение коэффициента теплоотдачи наножидкости на основе оксида меди в цилиндрическом канале [Текст] / Д. В. Гузей, А. В. Минаков, В. Я. Рудяк, А. А. Дектерев // Письма в ЖТФ. - 2014. - Т.40, №5. - С. 34-42.

9. Wang $X$. Thermal conductivity of nanoparticle-fluid mixture [Text] / X. Wang, X. Xu, S. Choi // J. Thermophys. Heat Transfer. - 1999. - Vol.13, Issue 4. - P. 474-480.

10. Anoop, K.B. Rheological and flow characteristics of nanofluids: influence of electroviscous effects and particle agglomeration [Text] / K.B. Anoop, S. Kabelac, T. Sundararajan, S.K. Das. // J. Appl. Phys. - 2009. Vol.106, Issue 3. P. 0.34909 .

11. Murshed, S.M.S. Investigations of thermal conductivity and viscosity of nanofluids [Text] / S.M.S. Murshed, K.C. Leong, C. Yang. // Int. J. Therm. Sci.- 2008. - Vol.47, Iss. 5. - P. 560-568.

12. Murshed, S.M.S. Temperature dependence of interfacial properties and viscosity of nanofluids for dropletbased microfluidics [Text] / S.M.S. Murshed, S. Tan, N. Nguyen // Journal of Physics. - 2008. - Vol.41, Issue 8. P. 1-5. 
13. Chandrasekar M. Experimental investigations and theoretical determination of thermal conductivity and viscosity of $\mathrm{Al}_{2} \mathrm{O}_{3} /$ water nanofluid [Text]/ $\mathrm{M}$. Chandrasekar, S. Suresh, A. Chandra. // Exp. Therm. Fluid Sci. 2010. - Vol.34, Issue 2. - P. 210-216.

14. Timofeeva, E.V. Nanofluids for heat transfer: an engineering approach [Text] / E.V. Timofeeva, W. Yu, D.M. France, D. Singh, J.L. Routbort // Nanoscale Res. Lett. 2011. - Vol. 6, Issue 182. - P. 1-7.

15. Prasher, R. Measurements of nanofluid viscosity and its implications for thermal applications [Text] / R. Prasher, D. Song, J. Wang, P. Phelan. // Appl. Phys. Lett. - 2006. - Vol. 89, Issue 13. - P. 133108.

16. Chen, H. Rheological behavior of ethylene glycol based titania nanofluids [Text] / H. Chen, Y. Ding, Y. He, C. Tan. // Chem. Phys. Lett. - 2007. - Vol.444. - P. 333-337.

17. Chen, $\mathbf{H}$. Rheological behaviour of nanofluids [Text] / H. Chen, Y. Ding, C. Tan. // New J. Phys. - 2007. - Vol. 9, Issue 367 - P. 1-24.

18. Kole, M. Viscosity of alumina nanoparticles dispersed in car engine coolant [Text] / M. Kole, T. Dey. // Exp. Therm. Fluid Sci. - 2010. - Vol.43, Issue 31 - P. 677-683.

19. Namburu, P. Experimental investigation of viscosity and specific heat of silicon dioxide nanofluids [Text] / P. Namburu, D. Kulkarni, A. Dandekar, D. Das. // Micro Nano Lett. - 2007. - Vol.2, Issue 3. - P. 67-71.

20. Namburu, P. Viscosity of copper oxide nanoparticles dispersed in ethylene glycol and water mixture [Text] / P. Namburu, D. Kulkarni, D. Misra, D. Das. // Exp. Therm. Fluid Sci. - 2007. - Vol. 32. - P. 397-402.

21. Garg, J. Enhanced thermal conductivity and viscosity of copper nanoparticles in ethylene glycol nanofluid [Text] / J. Garg, B. Poudel, M. Chiesa. // J. Appl. Phys. - 2008. Vol.103, Issue 7. - P. 1-6.

22. Nguyen, C. Temperature and particle-size dependent viscosity data for water-based nanofluids - hysteresis phenomenon [Text] / C. Nguyen, F. Desgranges, G. Roy. // Int. J. Heat Fluid Flow. - 2007. - Vol. 28, Issue 6. P. 1492-1506.

23. He, Y. Heat transfer and flow behaviour of aqueous suspensions of $\mathrm{TiO}_{2}$ nanoparticles (nanofluids) flowing upward through a vertical pipe [Text] / Y. He, Y. Jin, H. Chen // Int. J. Heat Mass Transfer. - 2007. - Vol. 50. P. 2272-2281.

24. Chevalier, J. Rheological properties of nanofluids flowing through microchannels [Text] / J. Chevalier, 0. Tillement, F. Ayela. // Appl. Phys. Lett. - 2007. - Vol. 91.
25. Pastoriza-Gallego, M. CuO in water nanofluid: influence of particle size and polydispersity on volumetric behavior and viscosity [Text] / M.Pastoriza-Gallego, C. Casanova, J. Legido, M. Piñeiro. // Fluid Phase Equilib. 2011. - Vol. 300. - P. 188-196.

26. Lu, W. Study for the particle's scale effect on some thermophysical properties of nanofluids by a simplified molecular dynamics method [Text] / W. Lu, Q. Fan. // Eng. Anal. Boundary Elem. - 2008. - Vol. 32. - C. 282-289.

27. Nguyen, $\mathbf{C}$. Viscosity data for $\mathrm{Al}_{2} \mathrm{O}_{3}$-water nanofluidhysteresis: is heat transfer enhancement using nanofluids reliable [Text] / C. Nguyen, F. Desgranges, N. Galanis // Int J. Therm. Sci. - 2008. - Vol. 47, Issue 2. - C. 103-111.

28. Zhelezny, V.P. A complex investigation of the nanofluids R600a-mineral oilAl $\mathrm{O}_{3}$ and R600a-mineral oil- $\mathrm{TiO}_{2}$. Thermophysical properties. [Text] / V.P. Zhelezny, N.N. Lukianov, O.Ya. Khliyeva, A. S. Nikulina, A.V. Melnyk // Int. J. Refrigeration. - 2008. - Vol. 74. - P. 486-502.

29. Никулин, А. Г. Экспериментальная установка для исследования процессов кипения чистых жидкостей и растворов в свободном объеме [Text] / А. Г. Никулин, Ю. В. Семенюк, Н. Н. Лукьянов. // Холодильна техніка і технологія. - 2013. - № 4(144). - С. 12-18.

30. Железный, В. П. Методические особенности экспериментального изучения процессов кипения нанофлюидов в свободном объеме [Text] / В. П.Железный, Ю. В. Семенюк, А. Г. Никулин, Н. Н. Лукьянов. // Вестник Международной академии холода. - 2014. - № 3. - C. 4-9.

31. Wang, R. Use of nanoparticles to make mineral oil lubricants feasible for use in a residential air conditioner employing hydro-fluorocarbons refrigerants [Text] / R. Wang, Q. Wu, Y. Wu. // Energy and Buildings. - 2010. Vol. 42, Issue 11. - P. 2111-2117.

32. Рудяк, В.я. Современное состояние исследований вязкости наножидкостей [Text] / В. Я. Рудяк. // Вестник НГУ. - 2015. - Т.10, вып. 1. - С. 5-22.

33. Xiang-Qi, W. A Review on Nanofluids - Part I: Theoretical and Numerical Investigations [Text] / W. Xiang-Qi, S. Arun. // Brazilian Journal of Chemical Engineering. - 2008. - Vol.25, Issue 4. - P. 613-630.

34. Meibo, X. Application of fullerene $C_{60}$ nano-oil for performance enhancement of domestic refrigerator compressors [Text] / X. Meibo, W. Ruixiang, Y. Jianlin. // Int. J. Refrigeration. - 2014. - Vol.40. - P. 398-403.

Отримана в редакції 10.01.2017, прийнята до друку 07.02.2017 


\title{
Density and viscosity of solutions refrigerant $\mathrm{R} 600 \mathrm{a}$ / mineral oil / fullerene $\mathrm{C}_{60}$
}

\author{
S. A. Moroz, N. N. Lukianov, V. P. Zhelezny \\ Odessa National Academy of Food Technologies, 112 Kanatna str., Odesa, 65039, Ukraine
}

\begin{abstract}
The results of an experimental study for the temperature and concentration dependencies of the density and viscosity of solutions of R600a / mineral oil HF16-12 / C60 fullerenes are presented in the paper. The density measurements were performed by a pycnometric method in the temperature range 258-353 $\mathrm{K}$. The viscosity was measured by the rolling ball method in the temperature range 253-283 $\mathrm{K}$. Based on obtained experimental data, the effect of admixtures of compressor oil and additives $C_{60}$ fullerenes on the density and viscosity of R600a was analyzed. It was shown that the additives of fullerenes contribute to lowering the viscosity of the working fluid (refrigerant R600a / mineral oil HF1612 ) in entire range of experimental parameters. The working fluid R600a / mineral oil HF16-12 / fullerenes $C_{60}$ is recommended for application in domestic refrigeration.
\end{abstract}

Keywords: Solutions; Refrigerant; Fullerenes; Experiment; Density; Viscosity; Concentration

\section{References}

1. Moroz, S.A., Khlieva, O.Ya., Lukianov, N.N., Zhelezny, V.P. (2016) The influence of the compressor oil viscosity and fullerenes $\mathrm{C60}$ additives in the oil on the energy efficiency of refrigeration compressor system. Vestnik of the International Academy of Refrigeration, No.41, 41-46 (in Russian). DOI: http://doi.org/10.21047/1606-4313-201616-1-41-46

2. Kedzierski, M.A. (2008) Effect of CuO Nanoparticle Concentration on R134a-Lubricant Pool Boiling Heat Transfer. Micro-Nanoscale Heat Transfer International Conference, 2008: Proceedings Taiwan, N. Y., 1-8. DOI: http://doi.org/10.1115/1.3072926

3. Azmi, W.H., Sharif, M.Z., Yusof, T.M. (2017) Potential of nanorefrigerant and nanolubricant on energy saving in refrigeration system - A review. Renew. Sust. Energ. Rev., Vol. 69, 415-428.

DOI: http://doi.org/10.1016/j.rser.2016.11.207

4. Wang, R. Wu Q., Wu Y. 2010. Use of nanoparticles to make mineral oil lubricants feasible for use in a residential air conditioner employing hydro-fluorocarbons refrigerants. Energy and Buildings, 42(11), 2111-2117. DOI: http://doi.org/10.1016/j.enbuild.2010.06.023

5. Chen, H. Ding Y., Tan C. 2007. Rheological behaviour of nanofluids. New Jornal of Physics, 9(10), 367. DOI: http://doi.org/10.1088/1367-2630/9/10/367

6. Zhu, D.S. Li X.F., Gao J.W., Wang X.J., Li H., Wu Z.J. (2007) Influence of SDBS on viscosity for aluminium nanosuspensions. International Congress of Refrigeration 2007, 1-7.

7. Tavman, I., Turgut, A., Chirtoc, M., Schuchmann, H.P., Tavman, S. (2008) Experimental investigation of viscosity and thermal conductivity of suspensions containing nanosized ceramic particles. International Scientific Journal, 34(2), 99-104.

8. Guzey, D.V., Minakov, A.V., Rudyak, V. Ya., Dekterev, A.A. (2014) Measurement of the heat transfer coefficient of a nanofluid on the basis of copper oxide in a cylindrical channel Letters in ZTF, 40(5), 34-42 (in Russian).
9. Wang, X., Xu, X., Choi, S. (1999) Thermal conductivity of nanoparticle-fluid mixture. J. Thermophys. Heat Transfer, 13(4), 474-480. DOI: https://doi.org/10.2514/2.6486 10. Anoop, K.B. Rheological and flow characteristics of nanofluids: influence of electroviscous effects and particle agglomeration [Text] / K.B. Anoop, S. Kabelac, T. Sundararajan, S.K. Das. // J. Appl. Phys. - 2009. Vol.106, Issue 3. P. 0.34909. doi: 10.1063/1.3182807

11. Murshed Sohel, S.M. Investigations of thermal conductivity and viscosity of nanofluids [Text] / S.M.S. Murshed, K.C. Leong, C. Yang. // Int. J. Therm. Sci.- 2008. - Vol.47, Issue 5. - P. 560-568. doi: 10.1016/j.ijthermalsci.2007.05.004

12. Murshed Sohel, S.M. Temperature dependence of interfacial properties and viscosity of nanofluids for droplet-based microfluidics [Text] / S.M.S. Murshed, S. Tan, N. Nguyen // Journal of Physics. - 2008. - Vol.41, Issue 8. P. 1-5.

13. Chandrasekar M. Experimental investigations and theoretical determination of thermal conductivity and viscosity of $\mathrm{Al}_{2} \mathrm{O}_{3} /$ water nanofluid [Text]/ M. Chandrasekar, S. Suresh, A. Chandra. // Exp. Therm. Fluid Sci. 2010. - Vol.34, Issue 2. - P. 210-216. DOI: 10.1016/j.expthermflusci.2009.10.022

14. Timofeeva, E.V. Nanofluids for heat transfer: an engineering approach [Text] / E.V. Timofeeva, W. Yu, D.M. France, D. Singh, J.L. Routbort // Nanoscale Res. Lett. 2011. - Vol. 6, Issue 182. - P. 1-7. DOI: 10.1186/1556276X-6-182.

15. Prasher, R. Measurements of nanofluid viscosity and its implications for thermal applications [Text] / R. Prasher, D. Song, J. Wang, P. Phelan. // Appl. Phys. Lett. - 2006. - Vol. 89, Issue 13. - P. 133108. doi: 10.1063/1.2356113 16. Chen, H. Rheological behaviour of ethylene glycol based titania nanofluids [Text] / H. Chen, Y. Ding, Y. He, C. Tan. // Chem. Phys. Lett. - 2007. - Vol.444. - P. 333-337. doi: 10.1016/j.cplett.2007.07.046

17. Chen, H. Rheological behaviour of nanofluids [Text] / H. Chen, Y. Ding, C. Tan. // New J. Phys. - 2007. - Vol.9, Issue 367 - P. 1-24. doi: 10.1088/1367-2630/9/10/367 
18. Kole, M. Viscosity of alumina nanoparticles dispersed in car engine coolant [Text] / M. Kole, T. Dey. // Exp. Therm. Fluid Sci. - 2010. - Vol.43, Issue 31 - P. 677-683. doi: 10.1016/j.expthermflusci.2009.12.009

19. Namburu, P. Experimental investigation of viscosity and specific heat of silicon dioxide nanofluids [Text] / P. Namburu, D. Kulkarni, A. Dandekar, D. Das. // Micro Nano Lett. - 2007. - Vol.2, Issue 3. - P. 67-71. doi: 10.1049/mnl:20070037

20. Namburu, P. Viscosity of copper oxide nanoparticles dispersed in ethylene glycol and water mixture [Text] / P. Namburu, D. Kulkarni, D. Misra, D. Das. // Exp. Therm. Fluid Sci. - 2007. - Vol. 32. - P. 397-402. DOI: 10.2298/TSCI140616025K

21. Garg, J. Enhanced thermal conductivity and viscosity of copper nanoparticles in ethylene glycol nanofluid [Text] / J. Garg, B. Poudel, M. Chiesa. // J. Appl. Phys. - 2008. Vol.103, Issue 7. - P. 1-6. DOI: 10.1063/1.2902483

22. Nguyen, C. Temperature and particle-size dependent viscosity data for water-based nanofluids - hysteresis phenomenon [Text] / C. Nguyen, F. Desgranges, G. Roy. // Int. J. Heat Fluid Flow. - 2007. - Vol. 28, Issue 6. - P. 1492-1506. doi: 10.1016/j.ijthermalsci.2008.03.009

23. He, Y. Heat transfer and flow behaviour of aqueous suspensions of $\mathrm{TiO}_{2}$ nanoparticles (nanofluids) flowing upward through a vertical pipe / Y. He, Y. Jin, H. Chen // Int. J. Heat Mass Transfer. - 2007. - Vol. 50. - P. 22722281. doi: 10.1016/j.ijheatmasstransfer.2006.10.024

24. Chevalier, J. Rheological properties of nanofluids flowing through microchannels [Text] / J. Chevalier, 0. Tillement, F. Ayela. // Appl. Phys. Lett. - 2007. - Vol. 91. doi: http://dx.doi.org/10.1063/1.2821117

25. Pastoriza-Gallego, M. CuO in water nanofluid: influence of particle size and polydispersity on volumetric behaviour and viscosity [Text] / M.Pastoriza-Gallego, C. Casanova, J. Legido, M. Piñeiro. // Fluid Phase Equilib. 2011. - Vol. 300. - P. 188-196. DOI: 10.1186/1556-276X6-221

26. Lu, W. Study for the particle's scale effect on some thermophysical properties of nanofluids by a simplified molecular dynamics method [Text] / W. Lu, Q. Fan. // Eng. Anal. Boundary Elem. - 2008. - Vol. 32. - C. 282-289. doi: 10.1016/j.enganabound.2007.10.006
27. Nguyen, $\mathrm{C}$. Viscosity data for $\mathrm{Al}_{2} \mathrm{O}_{3}$-water nanofluidhysteresis: is heat transfer enhancement using nanofluids reliable [Text] / C. Nguyen, F. Desgranges, N. Galanis // Int J. Therm. Sci. - 2008. - Vol. 47, Issue 2. - C. 103-111. DOI: 10.1016/j.ijthermalsci.2007.01.033

28. Zhelezny, V.P. A complex investigation of the nanofluids R600a-mineral oilAl $\mathrm{O}_{3}$ and $\mathrm{R} 600 \mathrm{a}$-mineral oil- $\mathrm{TiO}_{2}$. Thermophysical properties. [Text] / V.P. Zhelezny, N.N. Lukianov, O.Ya. Khliyeva, A. S. Nikulina, A.V. Melnyk // Int. J. Refrigeration. - 2008. - Vol. 74. - P. 486-502. doi: 10.1016/j.ijrefrig.2016.11.008

29. Nikulin, A.G. The experimental unit for investigation of pure liquids and solutions pool boiling process [Text] / A.G. Nikulin, Yu. V. Semenyuk, N.N. Lukianov. // Refrigeration technology and technology. - 2013. - Vol. 4, Issue 144. - P. 12-18. (in Russian).

30. Zhelezny, V.P. Methodical features of the experimental study of the processes of nanofluids pool boiling [Text] / V.P. Zhelezny, Yu. V. Semenyuk, A.G. Nikulin, N.N. Lukianov. // Vestnik of the International Academy of Refrigeration. - 2014. - Vol. 3. - P. 4-9. (in Russian).

31. Wang, R. Use of nanoparticles to make mineral oil lubricants feasible for use in a residential air conditioner employing hydro-fluorocarbons refrigerants [Text] / R. Wang, Q. Wu, Y. Wu. // Energy and Buildings. - 2010. Vol. 42, Issue 11. - P. 2111-2117.

doi: 10.1016/j.enbuild.2010.06.023

32. Rudyak, V.Ya. Modern status of researches of nanofluids viscosity [Text] / V. Ya. Rudyak. // Bulletin of NGU. - 2015. - Vol.10, Issue 1. - P. 5-22. (in Russian).

33. Xiang-Qi, W. A Review on Nanofluids - Part I: Theoretical and Numerical Investigations [Text] / W. Xiang-Qi, S. Arun. // Brazilian Journal of Chemical Engineering. - 2008. - Vol.25, Issue 4. - P. 613-630.

34. Meibo, X. Application of fullerene $C_{60}$ nano-oil for performance enhancement of domestic refrigerator compressors [Text] / X. Meibo, W. Ruixiang, Y. Jianlin. // Int. J. Refrigeration. - 2014. - Vol.40. - P. 398-403. DOI: 10.1016/j.ijrefrig.2013.12.004

Received 10 January 2017 Approved 07 February 2017 Available in Internet 17 March 2017 